\title{
Digitalisierung eines Weiterbildungsprogrammes für studentische Tutor*innen in Zeiten der Corona- Pandemie am Beispiel des Karlsruher Instituts für Technologie (KIT)
}

\author{
Patrizia Köhler, Verena Landgrebe, Franziska Nickolaus und Katrin Heß
}

\section{Zusammenfassung des Beitrags}

Der hier vorliegende Beitrag dokumentiert die pandemiebedingte Umstellung auf die Online-Lehre, diskutiert besondere Herausforderungen digitaler Transformation, reflektiert die daraus gewonnenen Erkenntnisse und gibt Ausblicke, wie neue Ideen und Konzepte nachhaltig im Tutor*innenprogramm am KIT etabliert werden können. Hierbei dienen die Evaluationsergebnisse aus dem Wintersemester 2020/21 als wesentlicher Impuls, um die Durchführung des Weiterbildungsprogramms zukunftsorientiert weiterzudenken.

Schlüsselbegriffe: (digitale) Lebrkompetenz • (a)synchrone Lebr-Lernangebote • Praxistransfer • Qualitätssicherung • kontinuierliche Weiterentwicklung

\section{Das Tutor*innenprogramm „Start in die Lehre“ am KIT}

Das Tutor*innenprogramm „Start in die Lehre“ unterstützt und begleitet seit 2007 angehende Tutor*innen am KIT bei ihrer Tätigkeit in der Lehre. Im Rahmen von „Start in die Lehre“ erweitern die studentischen Tutor*innen ihre Fachkompetenzen durch pädagogisch-didaktisches Wissen sowie reflektierte Lehrerfahrung, die im Dreiklang die Basis für die Entwicklung und den Ausbau von Lehrkompetenz bilden (vgl. Olsson/Mårtensson/Roxå 2010). Seit jeher wird im Tutor*innenprogramm am KIT großer Wert auf fach- und formatspezifische Angebote sowie hohen Praxistransfer gelegt. Somit werden Inhalte und Struktur des Tutor*innenprogramms regelmäßig auf Grundlage von studentischen Feedbacks, Rückmeldungen der Fach- bzw. Übungsleiter*innen sowie den Erfahrungen der Programmverantwortlichen überprüft und ggf. angepasst. Die letzte umfassende Programmanpassung vor Ausbruch der Corona-Pandemie fand im Sommersemester 2018 statt. 


\section{Aufbau des Tutor*innenprogramms ab dem Sommersemester 2018}

Das Tutor*innenprogramm „Start in die Lehre“ ist für die Dauer von einem Semester angelegt und startet noch vor offiziellem Vorlesungsbeginn mit einem ersten Workshop zu den Grundlagen. Hier lernen die Tutor*innen wichtige pädagogische und didaktische Grundlagen kennen, die relevant für die Planung, Gestaltung und Durchführung von Tutorien sind. Demzufolge wurden die folgenden Lernziele für den Grundlagenworkshop formuliert:

Die Teilnehmenden können:

- Tutorien planen und strukturieren, d. h. eine detaillierte Planung unter Berücksichtigung verschiedener Faktoren und Strategien für ihr Tutorium erstellen.

- Fachinhalte aufbereiten und präsentieren, d. h. die theoretischen Grundlagen des Lernens beschreiben und für die Aufbereitung und Präsentation von Fachinhalten anwenden.

- Lernen anregen und begleiten, d. h. Möglichkeiten zur Unterstützung und Begleitung von Lernen in ihrem Tutorium benennen und konkrete Maßnahmen für den Einsatz entwickeln.

- herausfordernde Situationen lösen, d. h. Handlungsstrategien zum Umgang mit schwierigen Lehr- und Lernsituationen im Tutorium entwickeln und anwenden.

Aufbauend auf diesen Grundlagen wählen die Tutor*innen aus einem breiten Angebot zwei weitere fach- und formatspezifische Workshops aus. In der Regel können die Tutor*innen frei wählen, jedoch werden gemeinsam mit den jeweiligen Fach- bzw. Übungsleiter*innen Empfehlungen erarbeitet, welche Vertiefungen sich für die jeweiligen Fächer und Formate besonders eignen.
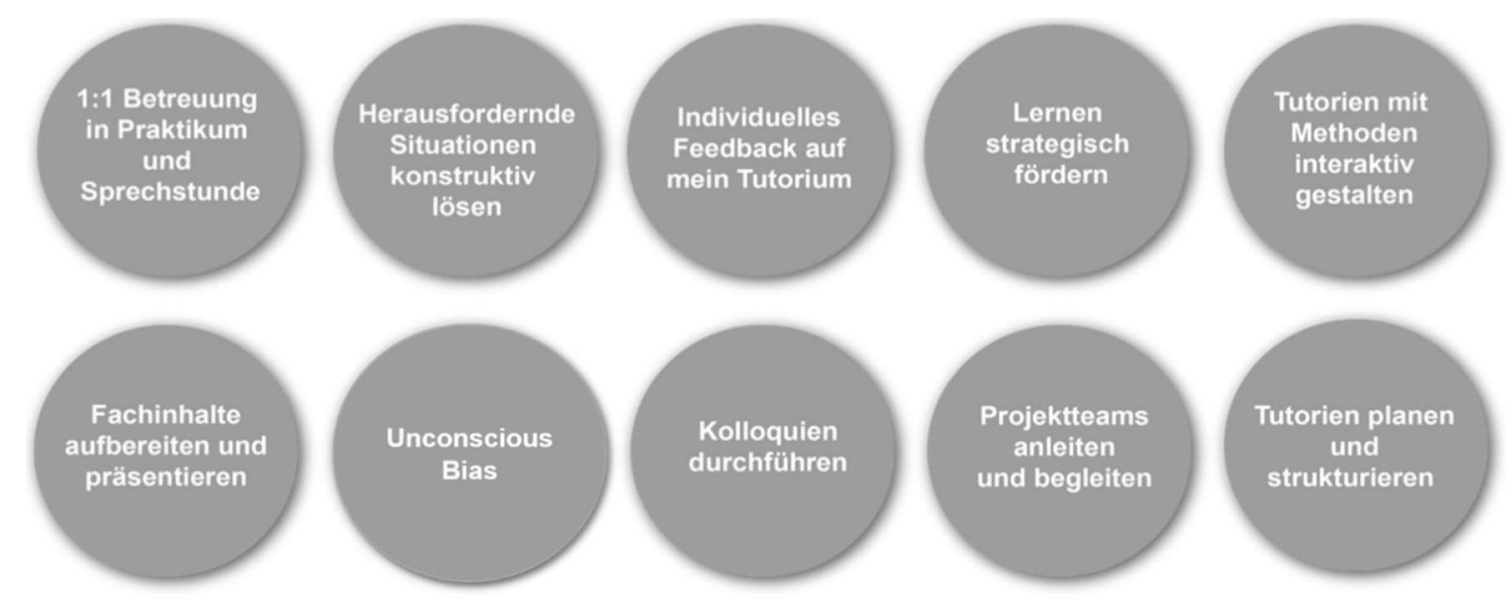

Abbildung 1: Übersicht über das Vertiefungsangebot

Ergänzend zu den drei (Präsenz-) Workshops, erstellen die Studierenden ein begleitendes Reflexionsportfolio (vgl. Baumgartner 2012). Dieses dient neben der Reflexion der absolvierten Workshops insbesondere der Stärkung des Praxistransfers, da die Studierenden im Rahmen 
von Reflexionsaufgaben aktiv dazu aufgefordert werden, Anwendungsmöglichkeiten des Gelernten für ihre eigenen Tutorien zu identifizieren und gleichfalls ihre bereits gesammelten Lehrerfahrungen in Bezug zu den Lernzielen des Workshops zu setzen (vgl. Heß et al. 2017). Insgesamt besteht das Reflexionsportfolio aus drei Reflexionsaufgaben, die sich jeweils unmittelbar auf die Grundlagen sowie die gewählten Vertiefungen beziehen und einer abschließenden Reflexionsarbeit mit Peer Review, die eine Art Retrospektive auf die Tutor*innentätigkeit im gesamten Semester darstellt. Das Reflexionsportfolio wird als E-Portfolio über das Integrierte Lern-, Informations- und Arbeitskooperations-System ILIAS angeboten. Somit handelt es sich bei „Start in die Lehre“ um ein Angebot, das auf einem Blended-Learning-Ansatz - im Sinne eines klassischen Rotationsmodells (vgl. Handke/Schäfer 2012; Christensen/Horn/Staker 2013) mit eindeutigem Schwerpunkt auf Präsenzveranstaltungen - basiert. Die gezielte Verzahnung von Präsenz- und Online-Phasen trägt zur zusätzlichen Steigerung des Praxistransfers bei. Beispielsweise lernen die Tutor*innen in den Präsenzveranstaltungen verschiedene didaktische Methoden kennen. Parallel zur begleitenden Online-Phase erproben sie diese in ihren Tutorien und dokumentieren bzw. reflektieren deren Einsatz (vgl. Heß et al. 2015).

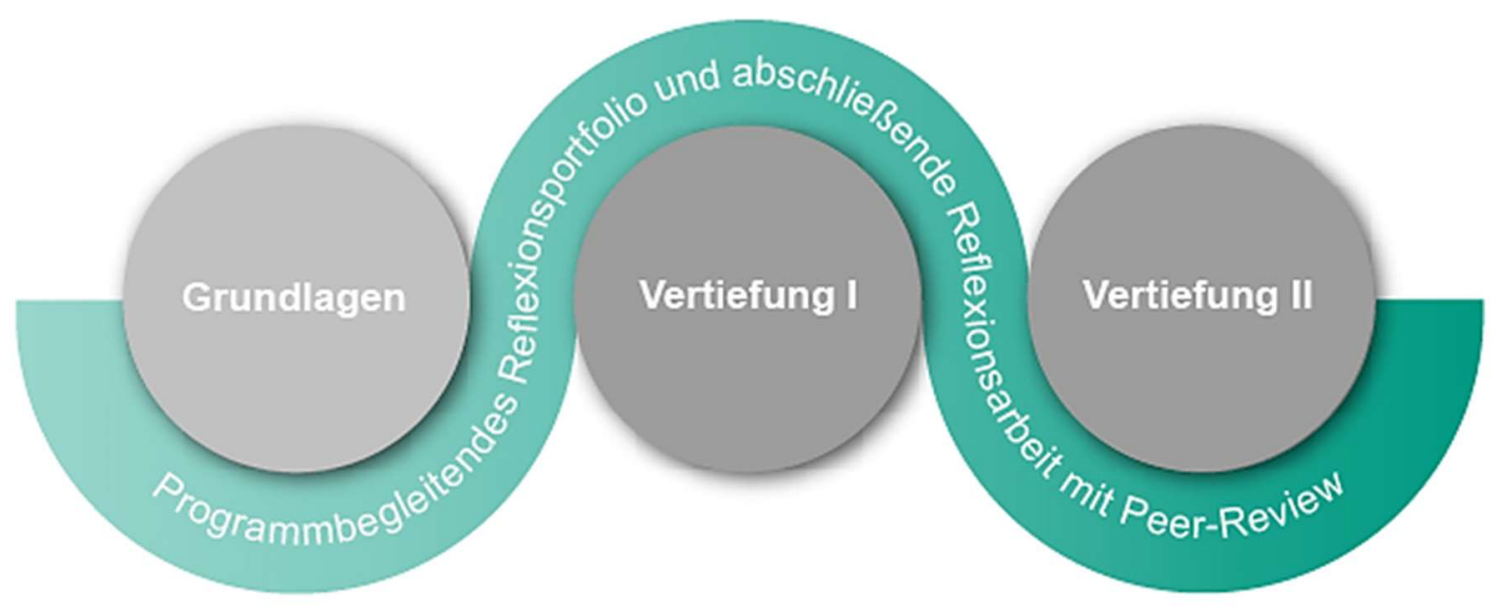

Abbildung 2: Programmablauf des Tutor*innenprogramms „Start in die Lebre“

\section{Durchfübrung des Tutor innenprogramms im Sommersemester 2020 (Corona-Semester)}

Mit Beginn des ersten Corona-Lockdowns am 22. März 2020 und der damit bestehenden Unsicherheit, ob das kommende Sommersemester 2020 unter gewohnten Bedingungen überhaupt stattfinden kann, entschlossen sich die Programmverantwortlichen von „Start in die Lehre“, eine Online-Alternative für die bereits für Ende März bis Mitte April 2020 geplanten Grundlagenworkshops zu erarbeiten. Dies stellte sich rückblickend als gute Entscheidung heraus, da das gesamte Semester (und auch die folgenden Semester) ausschließlich online stattfinden sollten. Innerhalb der nächsten vier Wochen arbeitete das verantwortliche Team unter Hochdruck an einem Online-Ersatzangebot für die Grundlagen, sodass dieses am 17. April 2020 für rund 80 angehende Tutor*innen starten konnte. Das Ersatzangebot zu den Grundlagen wurde als rein asynchrones Lernangebot über die Lernplattform ILIAS realisiert. Ein synchrones Angebot schien zum gegenwärtigen Zeitpunkt zu unsicher, da die Bereitstellung einer 
verlässlichen Infrastruktur für synchrone Lehr- und Lernangebote erst zum Vorlesungsstart, der pandemiebedingt auf den 04 . Mai 2020 verlegt wurde, zugesichert wurde.

Trotz fehlender synchroner Kommunikationsmöglichkeiten konnte das methodisch vielfältige Ersatzangebot über verschiedene ILIAS-Lernobjekte - wie beispielsweise Videos, Quizze, Übungen, Wikis, Foren und Lernmodule - gestaltet werden. Insgesamt konnten die Inhalte aus den Grundlagen im Rahmen von sechs Online-Modulen so aufbereitet werden, dass alle Lernziele im Sinne des Constructive Alignment (vgl. Biggs/Tang 2011) aus dem Präsenzworkshop theoretisch auch online erreicht werden konnten.

Zum Start der Grundlagenmodule stand bei vielen Tutor ${ }^{*}$ innen noch nicht fest, in welcher Form ihre Tutorien stattfinden würden. Selbst diejenigen, die bereits sicher wussten, dass sie ihre Tutorien online anbieten würden, hatten noch wenige Informationen darüber, wie diese sich konkret gestalten sollten und welche Online-Tools dafür zur Verfügung stehen würden. Diesem Umstand wurde zum einen dadurch Rechnung getragen, dass die Tutor*innen im Rahmen der ILIAS-Grundlagenmodule an passenden Stellen dazu aufgefordert wurden, das Gelernte auf die Online-Lehre zu übertragen. Beispielsweise wurde im Modul „Tutorien gestalten" im Wiki bzw. Forum gemeinsam diskutiert, welche der vorgestellten Methoden sich auch für die Online-Lehre eignen. Um auf Neuerungen im Tutorienbetrieb ad hoc reagieren und die Tutor*innen entsprechend mit adäquaten Informationen und Materialien versorgen zu können, wurde zum anderen ein Forum für „Fragen zur Online-Lehre“ im ILIAS-Kurs zu „Start in die Lehre" eingerichtet und begleitet.

Im weiteren Verlauf des Semesters musste auch ein Ersatzangebot für die Vertiefungen geschaffen werden. Seit Beginn der Vorlesungszeit standen mit Microsoft Teams und Zoom nun auch synchrone Kommunikationstools zur Gestaltung der Lehre zur Verfügung, sodass die Vertiefungen in einem synchronen Format realisiert werden konnten. Dabei wurden viele der mehrfach angebotenen Vertiefungen sowohl über Microsoft Teams als auch über Zoom durchgeführt, damit die Tutor*innen im Sinne des „didaktischen Doppeldeckers“ an einer Vertiefung teilnehmen konnten, die über das Kommunikationstool angeboten wurde, das sie auch selbst in ihrer eigenen Lehre einsetzen konnten.

Für die weiteren Bestandteile des Tutor*innenprogramms (Reflexionsportfolio inkl. Reflexionsarbeit und Peer Review) waren nur kleine inhaltliche Anpassungen notwendig, da diese bereits vor dem Corona-Semester im Rahmen eines Blended-Learning-Konzepts auch online gestaltet wurden.

\section{Herausforderungen bei der Überführung des ursprünglichen Programms in ein Online-Format}

Mit dem Start des Sommersemesters 2020 als erstes Online-Semester am KIT ging innerhalb weniger Wochen die Transformation einer auf Präsenzunterricht ausgerichteten Lehre in eine reine Online-Lehre einher. Die Programmverantwortlichen konnten daher nur auf ein kleines Repertoire an bestehenden Lernangeboten, wie Lehrvideos, zurückgreifen. Auch Lernplattformen und Videokonferenzsysteme wurden zu diesem Zeitpunkt bislang nur in Einzelfällen und als Ergänzung zum klassischen Präsenzunterricht eingesetzt. 


\section{Erböbter Beratungs- und Unterstützungsbedarf}

Für die Verantwortlichen des Tutor*innenprogramms galt es, neben der Überführung des semesterbegleitenden Weiterbildungsprogramms in ein Online-Format, ebenso dem erhöhten Beratungs- und Unterstützungsbedarf nachzukommen: Dieser betraf einerseits die Tutor*innen sowie andererseits die Lehrpersonen, die z. B. als Übungsleiter*innen für die grundlegende Gestaltung der Tutorienangebote der jeweiligen Studiengänge verantwortlich sind. Die Übungsleiter*innen waren gefordert, die gänzlich unterschiedlichen Tutoriumsformate, wie beispielsweise das Tafeltutorium, Rechnerpraktikum oder Kolloquium, in passgenaue, digitale Lehrszenarien zu überführen, sodass die Lehrangebote und -inhalte für die verschiedenen Zielgruppen und Bedarfe weiterhin vollumfänglich vorgefunden werden konnten. Auch auf Seiten der Tutor*innen veränderte sich der Unterstützungsbedarf beträchtlich. Als besonders herausfordernd erwiesen sich für die Teilnehmenden die neuen methodisch-didaktischen Anforderungen bei der Gestaltung von Online-Tutorien, zumal die Tutor*innen auf keine eigenen Erfahrungen in ihrem bisherigen Studium zurückgreifen konnten.

\section{Abbildung grundlegender Programminformationen und Lerninbalte in einem asynchronen Online-Format}

Durch den Wegfall des ursprünglich eintägigen Grundlagenworkshops in Präsenz, in dem rund 50 Teilnehmende alle relevanten Informationen über den Programmaufbau erhalten, sie sich das grundlegende Wissen für ihre neue Tätigkeit als Tutor*in aneignen und sich am Ende des Workshoptages gut vorbereitet für die Gestaltung der ersten Tutorien fühlen (sollten), standen die Programmverantwortlichen nun vor der Aufgabe, diesen elementaren Bestandteil des Weiterbildungsprogramms in ein asynchrones Online-Format zu überführen.

Für die Aufbereitung der Inhalte stand die Lernplattform ILIAS zur Verfügung. ILIAS wurde zuvor überwiegend als Reflexionsportfolio sowie für die Bereitstellung von Arbeitsmaterialien, weniger für die Nutzung asynchroner Lernformate verwendet. Die Kenntnisse zur technischen Gestaltung von digitalen Lernmodulen, Audio-Präsentationen, Quizzes und Foren mussten sich die Programmverantwortlichen in kurzer Zeit aneignen, wobei sie immer wieder auf technische Schwierigkeiten bei der Umsetzung stießen, wie beispielsweise das Hochladen von Videos im OpenCast-Format.

\section{Orientierung im Online-Format}

Die anschließende Evaluation der Grundlagen zum Ende des Sommersemesters 2020 zeigte auf, dass es den Teilnehmenden trotz der Nutzung verschiedener Informationskanäle, wie eines Fragenforums, FAQs in ILIAS und dem Versenden regelmäßiger Informationsmails, schwerfiel, die Anforderungen des Programms im Online-Format zu erfüllen. Eine Herausforderung für die Studierenden war dabei beispielsweise, den Überblick über die Bestandteile des Programms und deren Abgabefristen zu behalten. Auch Blaser und Graf (vgl. 2020) weisen darauf hin, wie wichtig eine eindeutige Formulierung der Aufgabenstellung, die klare Angabe von Bearbeitungsfristen sowie die Betonung der Verbindlichkeit bei der Aufgabenerfüllung für die asynchrone Informationsvermittlung sind. Für die Programmverantwortlichen äußerte 
sich dies vor allem in Form zahlreicher E-Mails und Anrufe mit Rückfragen zum weiteren Vorgehen. Zudem wurden vermehrt Abgabefristen für Einzelpersonen erweitert sowie kurzfristig Zusatzworkshops mit oft geringer Teilnehmendenzahl initiiert, um den Tutor*innen aufgrund der veränderten Rahmenbedingungen des Online-Formates den erfolgreichen Programmabschluss zu ermöglichen.

Aus diesen Gründen entschieden die Programmverantwortlichen, die asynchronen Lernmodule des Grundlagenbausteins um einen zweistündigen Online-Grundlagenworkshop im Wintersemester 2020/21 zu ergänzen. In diesem waren das Klären von offenen Fragen bezüglich des erfolgreichen Absolvierens sowie die detaillierte Besprechung des Programmablaufs wichtige Bestandteile. Ferner erhielten die Tutor*innen im Workshop die Gelegenheit, mit ihren Kolleg*innen in einen tiefergehenden Erfahrungsaustausch zu treten, erste Inhalte einzuüben, praktisch umzusetzen und sich wechselseitig Feedback darauf zu geben.

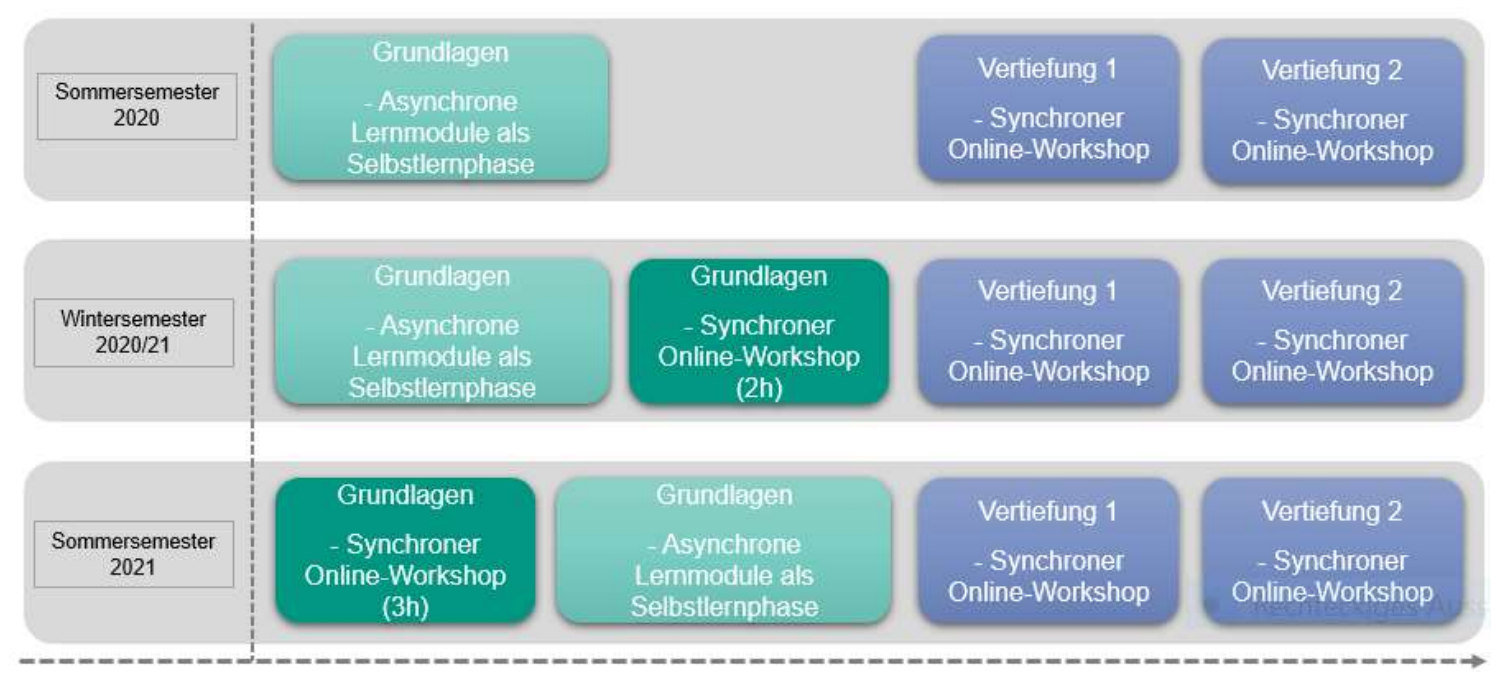

Abbildung 3: Überblick über die Änderungen im Programmablauf seit dem Sommersemester 2020

\section{Verwendung von Videokonferenzsystemen}

Bereits im Sommersemester 2020 wurde, als Ergänzung zu den asynchronen Lernangeboten des Grundlagenbausteins, auf die vom KIT bereitgestellten Videokonferenzsysteme Microsoft Teams und Zoom zurückgegriffen und es wurden synchrone Online-Vertiefungsworkshops initiiert. Das Ziel der Online-Workshops war vor allem, den persönlichen Erfahrungsaustausch unter den Tutor*innen zu fördern, da dieser aufgrund der Umstellung auf die asynchronen Grundlagenlernmodule nur sehr eingeschränkt in Form von Forumsbeiträgen möglich war. Die interaktive Gestaltung der Online-Workshops gelang aus Sicht der Programmverantwortlichen am besten mithilfe von Zoom, da dieses System zum einen die schnelle Einrichtung von Kleingruppen-Räumen, den so genannten „Breakout Sessions“, ermöglicht und zum anderen zusätzliche Kooperationstools, wie beispielsweise das Whiteboard oder die LiveUmfrage, beinhaltet. Trotz dieser Vorteile zeigte sich insbesondere zu Beginn der Online-Umstellung bei den Tutor*innen Verunsicherung und Skepsis gegenüber der Nutzung von Zoom, 
sodass manche der Teilnehmenden es ablehnten, ihre Kamera- und Mikrofonfunktion einzuschalten und dadurch lediglich eine aktive Beteiligung über das Versenden von Chatnachrichten möglich war. Auf Nachfrage begründeten sie dies mit der Sorge um den Schutz ihrer Daten.

\section{Technische Herausforderungen}

Zu Beginn der ersten Online-Workshops stellte sich sowohl für die Referent*innen als auch für die Teilnehmenden der Umgang mit der neuen technischen Umgebung als größtes Lernfeld heraus. Nach der Durchführung von zwei bis drei Online-Workshops minimierten sich die technischen Probleme, wie beispielsweise das Einrichten des Mikrofones und der Kamera oder das Teilen von Dokumenten, jedoch rasch beziehungsweise gelang die schnelle Beseitigung dieser. Insgesamt galt es, mehr Bearbeitungszeit insbesondere für die interaktiven Sequenzen mit den Teilnehmenden anzusetzen, da es vor den jeweiligen Aufgabenstellungen einer kurzen technischen Einführung in die verschiedenen Kooperationstools sowie Hilfestellung bei der Anwendung dieser bedurfte.

Indem die Teilnehmenden die Möglichkeit hatten, live mitzuerleben, wie die Referent ${ }^{*}$ innen die Online-Workshops technisch und methodisch-didaktisch gestalteten, erhielten sie wertvolle Anregungen für die eigene Durchführung der Online-Tutorien. Dieser sogenannte „didaktische Doppeldecker“, bei dem die behandelten Lerngegenstände sich mit den entsprechenden Lehrund Lernaktivitäten decken und so in der eigenen Erfahrung verankert werden (vgl. Wahl 2013), förderte die Nachhaltigkeit der individuellen Lernprozesse der Teilnehmenden.

Nach Einschätzung der Programmverantwortlichen stieg dadurch sowohl deren eigene digitale Kompetenz, als auch die Kompetenz der Tutor*innen, die fortan gefordert waren OnlineLehre zu gestalten. Unter digitaler Kompetenz werden hier - in Anlehnung an den Diskurs zur digital literacy im angelsächsischen Raum - Fähigkeiten verstanden, „which fit an individual for living, learning and working in a digital society“ (JISC 2014, o. S.). Angesichts des digitalen Wandels gehören diese Kompetenzen zu den Grundkompetenzen, die zu entwickeln sind, und die „Forderung, Lernende zum digitalen Kompetenzerwerb zu befähigen, gilt für Schul- und Hochschulbildung gleichermaßen“ (Bils/Brand/Pellert 2019, S. 1).

\section{Evaluationsinstrumente zur Qualitätssicherung und Weiterentwicklung des Weiter- bildungsprogramms}

Mit Blick auf das kommende Wintersemester 2021/22 wird das Weiterbildungsprogramm vorerst weiterhin online geplant und durchgeführt. Die bisherigen Erfahrungen bei der Überführung des ursprünglichen Programms in ein Online-Format legen die Fokussierung auf eine kontinuierliche Qualitätssicherung nahe. Aus diesem Grund sollen in einem nächsten Schritt Anhaltspunkte zur Weiterentwicklung des Online-Formats identifiziert werden. Als Evaluationsformat wurde auf quantitativer Ebene ein standardisierter Fragebogen sowie auf qualitativer Ebene eine Analyse der Reflexionsarbeiten der Tutor*innen aus dem Wintersemester 2020/21 herangezogen. Als ein zusätzliches Erhebungsinstrument sind qualitative Gruppeninterviews mit Tutor*innen für das Sommersemester 2021 geplant.

Der standardisierte Fragebogen wurde den Tutor*innen über ILIAS als Online-Umfrage am 
Ende des Wintersemesters 2020/21 bereitgestellt. Er umfasst 23 Items zum Gesamtprogramm, unterteilt in die Themenfelder Programm und-Bestandteile, Lernobjekte auf ILIAS, Inbalte, Arbeitsaufträge, Technik und Umsetzung sowie Lebrkompetenzentwicklung. Bei den geschlossenen Fragen werden den Tutor*innen vorwiegend fünf Antwortkategorien, beispielsweise von „trifft überhaupt nicht zu“ bis „trifft völlig zu“, sowie eine Ratingskala von 1-10 zur Verfügung gestellt. Der Fragebogen schließt mit zwei offenen Fragen ab. An der freiwilligen und anonymen Online-Befragung nahmen 24 von 148 Tutor*innen teil, die das Tutor*innenprogramm erfolgreich absolviert hatten.

\begin{tabular}{|c|c|c|c|c|c|c|c|}
\hline \multicolumn{8}{|l|}{ Programm und -bestandteile } \\
\hline \multicolumn{8}{|c|}{ Wie stark hast du vom Tutor*innenprogramm (Gesamtveranstaltung) sowie seinen einzelnen Bestandteilen profitiert? } \\
\hline & & - & $\cdot$ & $=$ & + & ++ & \\
\hline $\begin{array}{l}\text { Tutor*innenprogramm } \\
\text { (Gesamtveranstaltung) }\end{array}$ & \multirow{5}{*}{$\begin{array}{l}\text { überhaupt } \\
\text { nicht } \\
\text { profitiert }\end{array}$} & $\mathrm{O}$ & 0 & $\bigcirc$ & O & O & \multirow{5}{*}{$\begin{array}{c}\text { sehr stark } \\
\text { profitiert }\end{array}$} \\
\hline Grundlagen (ILIAS) & & O & O & 0 & 0 & O & \\
\hline Grundlagen-Ergänzung (Online-Workshop) & & 0 & 0 & 0 & 0 & 0 & \\
\hline Vertiefungen & & 0 & 0 & 0 & 0 & 0 & \\
\hline Reflexionsportfolio & & 0 & 0 & 0 & 0 & 0 & \\
\hline
\end{tabular}

Abbildung 4: Auszug aus dem Online-Fragebogen auf ILIAS

Zur Anfertigung der Reflexionsarbeiten wurden den Tutor*innen Leitfragen zur Orientierung im Schreibprozess bereitgestellt. Diese Leitfragen lauten beispielsweise „Inwiefern konnte dich das Tutor*innenprogramm bei der Weiterentwicklung deiner Kompetenzen/deines Tutoriums unterstützen?“ und „Welche Veranstaltungen/Inhalte waren dabei besonders hilfreich?“. Die Reflexionsarbeiten wurden anhand einer qualitativen Inhaltsanalyse in Anlehnung an Mayring und Fenzl (vgl. 2019) ausgewertet.

Die qualitative Analyse fokussiert sich hierbei auf das direkte Feedback der 148 Tutor*innen zum Tutor*innenprogramm. Dazu wurden zunächst zwei Überkategorien („Feedback Tutor*innenprogramm“ und „Feedback Online-Lehre“) deduktiv aus den Leitfragen abgeleitet. Spezifischere Unterkategorien wurden induktiv aus den Themen der gefilterten Textstellen gebildet. Tabelle 1 bietet eine Übersicht über einige Kategorien mit beispielhaften Phrasen, die für die Weiterentwicklung des Tutor*innenprogramms von besonderer Bedeutung sind und auf die im folgenden Kapitel genauer eingegangen wird.

\begin{tabular}{|c|c|c|}
\hline KATEGORIE & DEFINITION & ANKERBEISPIEL \\
\hline $\begin{array}{l}\text { Didaktischer Dop- } \\
\text { peldecker }\end{array}$ & $\begin{array}{l}\text { Alle Textstellen, die auf das } \\
\text { Vorleben der zu vermittelnden } \\
\text { Thematiken durch die Refe- } \\
\text { rent*innen eingehen. }\end{array}$ & $\begin{array}{l}\text { „Vor allem in der online-Lehre [sic] boten einem die } \\
\text { verschiedenen Workshops Einblick in den Ablauf ei- } \\
\text { nes online-Gruppenmeetings [sic]. Dabei konnte ich } \\
\text { verschiedene Möglichkeiten der Gestaltung sehen, } \\
\text { welche für meine anstehenden ersten Tutorien hilf- } \\
\text { reich waren.“ }\end{array}$ \\
\hline Vertiefungen & Alle Textstellen, die positive o- & $\begin{array}{l}\text { "Insbesondere die Vertiefung Tutorien mit Metho- } \\
\text { den interaktiv gestalten war sehr hilfreich, um mir bei }\end{array}$ \\
\hline
\end{tabular}




\begin{tabular}{l|l|l}
\hline & $\begin{array}{l}\text { der negative Kritik zu be- } \\
\text { stimmten Vertiefungs- } \\
\text { workshops beinhalten. }\end{array}$ & der Anwendung dieser Methoden zu helfen.“ \\
\hline $\begin{array}{l}\text { Lehr-/Lern- } \\
\text { arrangement }\end{array}$ & $\begin{array}{l}\text { Alle Textstellen, die sich auf } \\
\text { Vor- und Nachteile synchro- } \\
\text { ner und asynchroner Lehr- } \\
\text { /Lernarrangements beziehen. }\end{array}$ & $\begin{array}{l}\text { "Besonders hilfreich waren die Online-Veranstaltun- } \\
\text { in dem [sic] man die Durchführung eines kurzen Kol- } \\
\text { loquiums üben kann und direkt ein Feedback be- } \\
\text { kommt.“ }\end{array}$ \\
\hline
\end{tabular}

Tabelle 1: Beispielkategorien aus den Reflexionsarbeiten (eigene Darstellung)

\section{Lessons Learned und Quintessenz aus den Rückmeldungen der Teilnehmenden}

Die Rückmeldungen der Teilnehmenden zum Tutor*innenprogramm fallen überwiegend positiv aus. Trotz einzelner Beiträge innerhalb der Reflexionsarbeiten, in denen der (vorübergehende) Verlust der Präsenzlehre bedauert wird, zeigt die Auswertung des Online-Fragebogens, dass die Studierenden der Umstellung tendenziell offen gegenüberstehen.

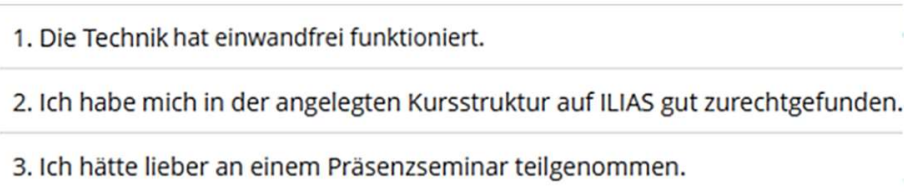

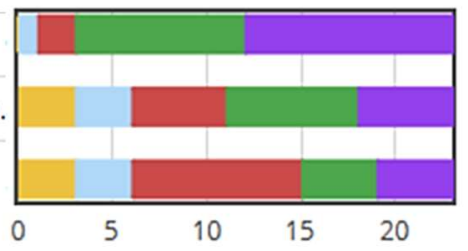

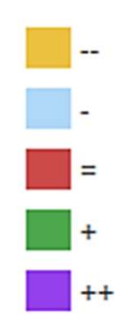

Abbildung 5: Auswertung zur Frage „Wie stark stimmst du den folgenden Aussagen zu?“ aus der Online-Umfrage des Wintersemesters 2020/21

Das übergeordnete Ziel, die angehenden Tutor*innen auf ihre Rolle als Lehrpersonen vorzubereiten, wurde erfüllt. Die Tutor*innen beantworteten die Frage „Wie gut fühlst du dich durch das Tutor*innenprogramm auf deine Aufgaben als Tutor*in vorbereitet?" auf einer Skala von 0 bis 10 - wobei 0 ,überhaupt nicht vorbereitet“ und 10 „sehr gut vorbereitet“ bedeutet - im Schnitt mit einem Wert von 7,8.

Wie gut fühlst du dich durch das Tutor*innenprogramm auf deine Aufgaben als Tutor*in vorbereitet?

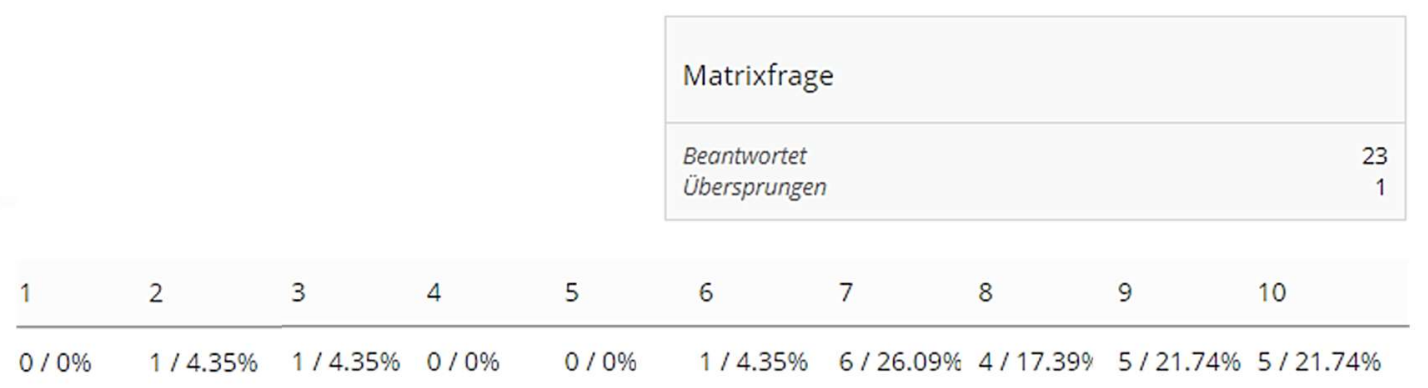

Abbildung 6: Auswertung zur Frage „Wie gut fühlst du dich durch das Tutor*innenprogramm auf deine Aufgabe als Tutor*in vorbereitet?“ aus der Online-Umfrage des Wintersemesters 2020/21 
Weiterhin lassen sich aus den Rückmeldungen der Teilnehmenden sowohl in Hinblick auf die Auswertung der Reflexionsarbeiten als auch des Online-Fragebogens deutliche Trends ablesen.

\section{Vertiefungen}

Insgesamt werden vor allem die synchronen Anteile des Tutor*innenprogramms positiv bewertet, insbesondere Vertiefungsworkshops wie „Fachinhalte aufbereiten und präsentieren“ und „Tutorien mit Methoden interaktiv gestalten“, in denen die Teilnehmenden einen Teil ihres Tutoriums vor Kommiliton*innen präsentieren und Methoden vorgestellt und ausprobiert werden. Eine Mehrheit der Teilnehmenden führt Simulationen mit Feedback sowie didaktische Methoden als hilfreich für ihre Online-Lehre an. Die Simulationen mit Feedback ermöglichen den Tutor*innen einen Einblick, wie sie vor dem Bildschirm auf ihre Tutand*innen wirken und welche Rolle, trotz oder gerade in der Online-Lehre, ihre Mimik und Gestik vor der Kamera spielt. Die aufbereiteten Methoden ermöglichen zudem vielen Teilnehmenden nach eigener Aussage, ihr Tutorium interaktiver zu gestalten und ihre Tutand*innen trotz der physischen Distanz zu motivieren. Eine Tutorin schreibt dazu, dass sie „es sehr spannend [fand], wie man durch den Einsatz von interaktiven Methoden die Tutanden länger motiviert und aktiv halten kann. Das habe [sie] bei [ihren] Workshops im Programm selbst bemerkt“. Im Hinblick auf einige Methoden wird allerdings auch Kritik geäußert, dass sich diese noch zu sehr auf die Präsenzlehre beziehen und nur bedingt auf die Online-Lehre übertragen ließen, wie beispielswiese die gemeinsame Erstellung eines Advance Organizers nach Ausubel (vgl. 1960).

\section{Didaktischer Doppeldecker}

Auch der von den Programmverantwortlichen wahrgenommene positive Effekt des „didaktischen Doppeldeckers“ wird durch das Feedback der Tutor*innen bestätigt. Teilnehmende, die sich in ihren Reflexionsarbeiten auf diesen Aspekt beziehen, heben vor allem positiv hervor, dass sie durch die synchronen Workshops bereits die Funktionen der Online-Plattformen sowie Ideen zur Gestaltung von Lehrveranstaltungen kennenlernen, an sich selbst erfahren und in ihren Tutorien umsetzen konnten. So schreibt ein Teilnehmer beispielsweise, dass er „im Grundlagenworkshop [...] damit vertraut [wurde], dass auf der Plattform „Zoom“, die auch für [sein] Onlinetutorium verwendet wurde, viele Möglichkeiten bestehen, die Teilnehmer interaktiv am Tutorium teilhaben zu lassen. “ und ein anderer Tutor ergänzt, dass „dadurch die Idee [entstand], mit Hilfe von Breakout-Rooms Gruppenarbeiten mit in das Tutorium einzubauen".

\section{Lehr-Lernarrangement}

Ein Vorteil der synchronen Veranstaltungen liegt laut dem Feedback aus den Reflexionsarbeiten außerdem in der Möglichkeit zum direkten Austausch mit anderen Tutor*innen, sowohl aus denselben wie auch aus anderen Fachrichtungen. Ein Tutor äußerte sich dazu folgendermaßen: „Der Austausch mit den Studierenden, aber auch die Möglichkeit zu haben, in COVID-19-Zeiten die Sozialkontakte zusammen mit Wissenszuwachs zu fördern, war für mich 
eine sehr positive Erfahrung.“. Die Interaktion mit Kommiliton*innen bietet demnach den doppelten Vorteil, sich sowohl persönlich als auch fachlich austauschen zu können und voneinander zu lernen.

Dass sich die synchronen Teile des Tutor*innenprogramms größerer Beliebtheit erfreuen als die asynchronen, bestätigt auch die Auswertung des Online-Fragebogens. Hierbei geben $59 \%$ der Teilnehmenden an, dass sie von dem Grundlagenworkshop stark oder sehr stark profitiert haben - bei den Vertiefungen sind es sogar knapp $83 \%$-, wogegen zur asynchronen Selbstlernphase auf ILIAS nur etwa $43 \%$ angeben, (sehr) stark von dieser profitiert zu haben.

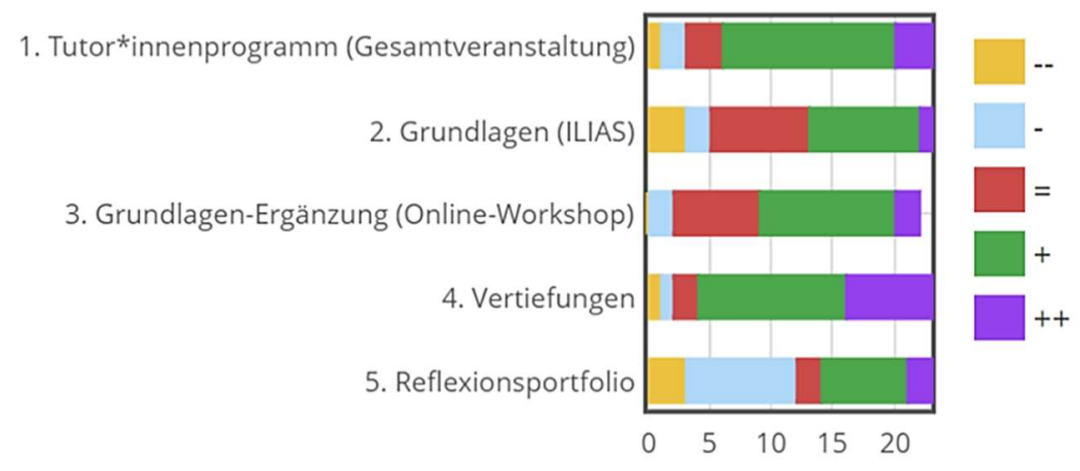

Abbildung 7: Auswertung zur Frage „Wie stark hast du vom Tutor*innenprogramm (Gesamtveranstaltung) sowie seinen einzelnen Bestandteilen profitiert?“ aus der Online-Umfrage des Wintersemesters 2020/21

Anregungen für die zukünftige Gestaltung

Aus diesen Erkenntnissen können für die zukünftige Durchführung des Tutor*innenprogramms einige wichtige Anregungen mitgenommen werden. Zum einen erweist es sich als sinnvoll, den Raum für Austausch zwischen den Tutor*innen sowie Simulationen und Übungen mit Peer-Feedback in den synchronen Veranstaltungen zu erweitern. Die theoretischen Hintergründe können ergänzend dazu vermehrt auf Vorbereitungs- und Selbstlernphasen auf ILIAS ausgelagert werden. Dies deckt sich mit den Einschätzungen von Blaser und Graf, wonach die asynchronen Phasen hauptsächlich dem Wissenserwerb und der Informationsvermittlung dienen, während die synchronen Phasen „zur Wissensvertiefung, Weiterentwicklung, Kollaboration und zum sozialen Kontakt genutzt werden“ (Blaser und Graf 2020, S. 3) können. Bei einer vermehrten Auslagerung der Theorie bleibt gleichzeitig zu beachten, dass die Selbstlernphasen nicht überfrachtet werden und die Teilnehmenden auch hier durch Wissenstests, Wikis, oder Ähnliches aktiviert werden (vgl. Blaser und Graf 2020). Dieser Notwendigkeit wurde in der bisherigen Ausgestaltung der Selbstlernphasen, unter anderem durch den Einsatz von Quizzes, Foren und Peer-Reviews, bereits weitreichende Beachtung geschenkt. Dennoch werden für das kommende Semester einzelne Lernphasen weiter überarbeitet, um beispielweise durch das Vertonen von Präsentationen die Theoriephasen persönlicher und vielfältiger zu gestalten. Weitergehend sollen auch zukünftig in den asynchronen Angeboten Möglichkeiten zum Austausch über Foren angeboten und ausgebaut werden. Der mehrfach geäußerten Kritik innerhalb der Reflexionsarbeiten und des Online-Fragebogens, dass Fragen und Anliegen in den Foren verspätet oder gar nicht beantwortet wurden, wird mit der klaren 
Einteilung von Zuständigkeiten innerhalb des Programmteams sowie der Zusammenfassung und Weiterverwendung der Forenbeiträge begegnet. Um die Übertragung der Methoden von der Präsenz- in die Online-Lehre zu erleichtern, sollen zudem die Methodenblätter mit Tipps zur Online-Umsetzung ergänzt werden. Hierfür erweist es sich als sinnvoll, den Teilnehmenden zusätzliche Online-Tools, wie Pingo KIT (pingo.scc.kit.edu) oder learningapps.org, zur Gestaltung von interaktiven Elementen an die Hand zu geben. Wie auch zu den Online-Plattformen sind dazu zunächst Recherchen zu Vor- und Nachteilen sowie den Datenschutzbedingungen der verschiedenen Tools notwendig.

\section{Erfolgsfaktoren und Hinweise zur Übertragbarkeit}

Nach eigener Einschätzung wurde die digitale Transformation des Tutor*innenprogramms durch vielfältige Faktoren bestimmt, die sich positiv auf den Erfolg des Transformationsprozesses auswirkten. An erster Stelle ist das erweiterte Programmteam zu nennen. Dieses unterstützte schon vor dem Sommersemester 2020 die Programmverantwortlichen bei der Durchführung des Tutor*innenprogramms, z.B. durch die Übernahme von Vertiefungsworkshops, und stellte zur Überführung in ein Online-Format kurzfristig zusätzliche personelle Ressourcen bereit. Ferner war die technische Affinität des Kernteams sowie der wöchentliche Austausch der Programmverantwortlichen in Qualitätszirkeln von großer Bedeutung. Hier wurde u.a. regelmäßig das Feedback der Tutor*innen aufgegriffen und zur Qualitätssicherung sowie kontinuierlichen Weiterentwicklung des Programms verarbeitet. Um die technische Anpassung des Weiterbildungsprogramms in ein Online-Format erfolgreich zu bewerkstelligen, war das Vorhandensein einer adäquaten Infrastruktur (z.B. Lernplattform, Videokonferenzsysteme) durch das Steinbuch Centre for Computing (Rechenzentrum des KIT) sowie das Angebot von Weiterbildungsmöglichkeiten für die Mitarbeitenden wie beispielsweise die externe Weiterbildung zum bzw. zur Onlinetrainer*in essentiell. Der bereits bestehende Blended-Learning-Ansatz sowie die Verwendung von bekannten Tools wie beispielsweise ILIAS erleichterte zusätzlich den Übergang sowie das Ankommen in der Online-Lehre. Diese Voraussetzungen bzw. Maßnahmen haben aus Sicht der Programmverantwortlichen maßgeblich dazu beigetragen, das Tutor*innenprogramm zu transformieren und lassen sich im Sinne von generalisierten Erfolgsfaktoren auf andere Weiterbildungsprogramme innerhalb des KIT und gegebenenfalls auch auf andere Hochschulen übertragen.

\section{Ausblick}

Insgesamt war die Umstellung des Tutor*innenprogramms von der Präsenz- in die OnlineLehre für alle Beteiligten mit vielfältigen Neuerungen und Herausforderungen verbunden. Dennoch werden mit der Etablierung von digitalen Weiterbildungsangeboten zunehmend deren Vorteile deutlich. Zum einen bietet die Online-Lehre die Möglichkeit, Ressourcen in materieller, räumlicher und personeller Hinsicht optimiert einzusetzen. Zum anderen wird den Studierenden der schnelle Zugang zu Lernangeboten und damit ihre Integration in den weiteren Studienalltag erleichtert. Eine Studie von Berghoff et al. aus dem Jahr 2021 bestätigt, dass sich sowohl Studierende als auch Lehrende auch für die Zukunft eine gezielte Einbindung digitaler Lehrelemente wünschen. 
In den vergangenen Semestern wurden die medialen und digitalen Kompetenzen sowohl bei den Programmverantwortlichen als auch bei den Studierenden am KIT erfolgreich ausgebaut und sollen auch weiterhin zukunftsorientiert genutzt und gestärkt werden. Dieses Vorhaben stützt auch die Aussage von Bildungsministerin Karliczek, die im Mai 2021 in einer Pressemitteilung die Vorreiterfunktion der Universitäten betonte, welche als „Innovationsschmiede digitaler Lehre" fungieren und auch bei einer Rückkehr zu mehr Präsenz neu gewonnene Ideen einsetzen sollen, um eine zeitgemäße Hochschullehre zu gestalten (vgl. BMBF 2021). Aus diesen Gründen ist eine bedarfsorientierte Beibehaltung der Online-Lehre im Tutor*innenprogramm des KIT auch in Zukunft abzuwägen. In welchem Ausmaß Elemente der OnlineLehre übernommen werden, ist unter anderem davon abhängig, in welchem Format die Zielgruppe ihre Tutorien in den nächsten Semestern durchführen wird. Sollten die Tutorien wieder zunehmend in Präsenz stattfinden, kann es sich als sinnvoll erweisen, auch die Weiterbildungselemente hauptsächlich in Präsenz anzubieten, um die Vorteile des didaktischen Doppeldeckers weiterhin optimal zu nutzen. Inwieweit Formate für Präsenz- bzw. Online-Lehre angeboten werden, hängt damit insbesondere von den Anforderungen des jeweiligen Fachbereichs und den sich ändernden Rahmenbedingungen ab.

Die Programmverantwortlichen betrachten rückblickend den Transformationsprozess von einer auf Präsenzunterricht ausgerichteten Lehre in eine Online-Lehre trotz der damit einhergehenden Herausforderungen als richtungsweisend für die digitale Kompetenzentwicklung der Lehrpersonen und Studierenden und werden diese in ihrem Weiterbildungsprogramm auch nach der Rückkehr zur Präsenzlehre weiter fokussieren.

\section{Literatur}

Ausubel, David Paul (1960): The use of advance organizers in the learning and retention of meaningful verbal material. In: Journal of Educational Psychology, 51, pp. 267-272.

Baumgartner, Peter (2012): Eine Taxonomie für E-Portfolios - Teil II des BMWF-Abschlussberichts "E-Portfolio an Hochschulen": GZ 51.700/0064-VII/10/2006.

Berghoff, Sonja/Horstmann, Nina/Hüsch, Marc/Müller, Katrin (2021): Studium und Lehre in Zeiten der Corona-Pandemie - Die Sicht von Studierenden und Lehrenden. Gütersloh: CHE Impulse.

Biggs, John/Tang, Catherine (2011): Teaching for Quality Learning at University: What the Student Does. Maidenhead: Open University Press.

Bills, Annabell/Brand, Heike/Pellert, Ada (2019): Hochschule(n) im digitalen Wandel. Bedarfe und Strategien. URL: bpb.de/apuz/293132/hochschulen-im-digitalen-wandel (04.08.2021).

Blaser, Daniela/Graf, David (2020): Online-Lehre - Tipps und Empfehlungen. Universität Bern, Hochschuldidaktik \& Lehrentwicklung. URL: boris.unibe.ch/id/eprint/145165 (26.05.2021).

Bundesministerium für Bildung und Forschung (BMBF) (2021): Karliczek: Hochschulen müssen Innovationsschmieden digitaler Lehre sein. URL: bmbf.de/bmbf/shareddocs/pressemitteilungen/ de/karliczek-hochschulen-muessen--schmieden-digitaler-lehre-sein.html (04.08.2021).

Christensen, Clayton M./Horn, Michael B./Staker, Heather (2013): Is K-12 Blended Learning Disruptive? An Introduction to the Theory of Hybrids. URL: christenseninstitute.org/publications/hyb rids (01.03.2021).

Handke, Jürgen/Schäfer, Anna (2012): E-Learning, E-Teaching und E-Assessment in der Hochschullehre. München: Oldenbourg Wissenschaftsverlag. URL: doi.org/10.1524/9783486716849. 
Heß, Katrin/Schostok, Patrizia/Klink, Karina/Diez, Anke (2015): Reflektierter Methodeneinsatz: Ein Instrument zur Entwicklung von fach- und formatspezifischer Lehrkompetenz. In: Behrendt, Brigitte (Hrsg.): Neues Handbuch Hochschullehre: Lehren und Lernen effizient gestalten. Berlin: Raabe, L 1.27, S. 133-158.

Heß, Katrin/Schostok, Patrizia/Klink, Karina/Diez, Anke (2017): Der Einsatz von E-Portfolios im Rahmen hochschuldidaktischer Weiterbildungsprogramme zur Förderung des Praxistransfers - am Beispiel des Tutorenprogramms am KIT. In: Kordts-Freudinger, Robert/Al-Kabbani, Daniel/Schaper, Niclas (Hrsg.): dghd Tagungsband 2015. „Hochschuldidaktik im Dialog“. Reihe Blickpunkt Hochschuldidaktik. Bielefeld: W. Bertelsmann Verlag, S. 183-194.

JISC (2014): Developing digital literacies. URL: jisc.ac.uk/full-guide/developing-digital-literacies (02.08.2021).

Mayring, Philipp/Fenzl, Thomas (2019): Qualitative Inhaltsanalyse. In: Baur, Nina/Blasius, Jörg(Hrsg.): Handbuch Methoden der empirischen Sozialforschung. Wiesbaden: Springer VS, S. 633-648.

Olsson, Thomas/Mårtensson, Katarina/Roxå, Torgny (2010): Pedagogical Competence - A Development Perspective from Lund University. In: Ryegard, Asa (Ed.): A Swedish Perspective on Pedagogical Competence. Unter Mitarbeit von Karin Apelgren und Thomas Olsson. Uppsala University, pp. 121-132.

Wahl, Diethelm (2013): Lernumgebungen erfolgreich gestalten. Vom trägen Wissen zum kompetenten Handeln. Bad Heilbrunn: Klinkhardt, S.64-69. 


\section{Informationen zu den Autorinnen}

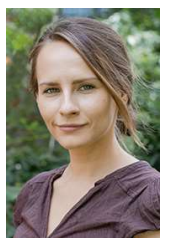

Patrizia Köhler (Dipl.-Päd.) ist Mitarbeiterin in der Personalentwicklung und Beruflichen Ausbildung am Karlsruher Institut für Technologie (KIT). Ihre Arbeitsschwerpunkte umfassen die Qualifizierung wissenschaftlicher Mitarbeiter*innen sowie das Weiterbildungsprogramm für Tutor*innen.

patrizia.koehler@kit.edu

Verena Landgrebe (Dipl.-Päd.) ist Mitarbeiterin in der Personalentwicklung und Beruflichen Ausbildung am Karlsruher Institut für Technologie (KIT). Ihre Arbeitsschwerpunkte umfassen Angebote zur Kompetenzentwicklung für wissenschaftliche Mitarbeiter*innen sowie das Weiterbildungsprogramm für Tutor*innen.

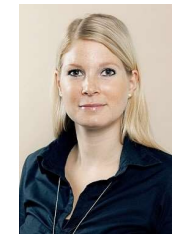

verena.landgrebe@kit.edu

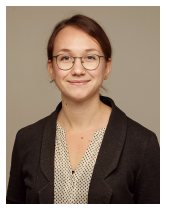

Franziska Nickolaus (Bildungswissenschaftlerin M.A.) ist Mitarbeiterin in der Personalentwicklung und Beruflichen Ausbildung am Karlsruher Institut für Technologie (KIT). Ihre Arbeitsschwerpunkte umfassen die Qualifizierung wissenschaftlicher Mitarbeiter*innen sowie das Weiterbildungsprogramm für Tutor*innen.

franziska.nickolaus@kit.edu

Katrin Heß (Dipl.-Inform.) ist Mitarbeiterin in der Personalentwicklung und Beruflichen Ausbildung sowie Leiterin der Arbeitsstelle für Hochschuldidaktik am Karlsruher Institut für Technologie (KIT). Ihre Arbeitsschwerpunkte umfassen das Baden-WürttembergZertifikat für Hochschuldidaktik sowie das Weiterbildungsprogramm für Tutor*innen.

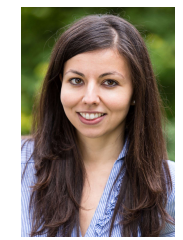

katrin.hess@kit.edu

\section{Zitationshinweis:}

Köhler, Patrizia/Landgrebe, Verena/Nickolaus, Franziska/Heß, Katrin (2021): Digitalisierung eines Weiterbildungsprogrammes für studentische Tutor*innen in Zeiten der Corona-Pandemie am Beispiel des Karlsruher Instituts für Technologie (KIT). In: Online-Magazin Ludwigsburger Beiträge zur Medienpädagogik, Ausgabe 21/2021. URL: medienpaed-ludwigsburg.de/ 